\title{
Laudatio anlässlich der Verleihung der DEGUM-Medaille an Prof. Dr. Wolfram Wermke
}

40. Dreiländertreffen 2016, Leipzig 27.10.2016

Mit Wolfram Wermke bin ich seit 1990 freundschaftlich verbunden und ich freue mich daher außerordentlich, dass er mit der DEGUM-Medaille für seine besonderen Verdienste um den Ultraschall ausgezeich- net wird und übernehme sehr gerne die Laudatio im Auftrag unseres Präsidenten und Sektionsleiters.

Ich werde nach seinem hervorragenden Vortrag, nachdem sich eine Würdigung sei- ner sonografischen Verdienste eigentlich erübrigt, einige seiner Charaktereigenschaften herausstellen, damit mich Wolfram hinterher nicht der Lobhudelei bezichtigen kann. 


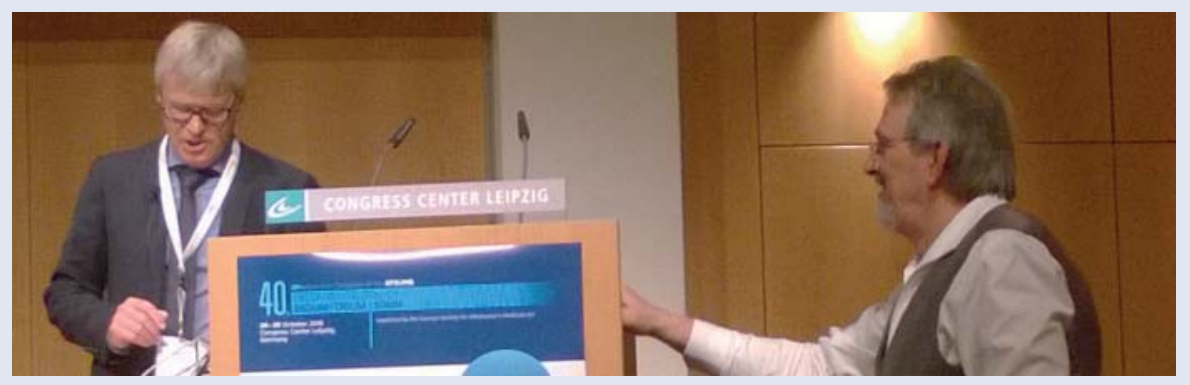

Schon vor der Wende waren mir seine Publikationen aufgefallen. Ich war neugierig auf den Typen hinter der Mauer, der so brilliant sononografisch arbeitete und dessen wissenschaftlichen Interessen mit meinen weitgehend übereinstimmten. Es ging damals in erster Linie um Gallengangsdiagnostik.

Wolfram Wermke publizierte ab 1985 reichlich bei der PubMed-gelisteten DDRZeitschrift für Ärztliche Fortbildung und ab 1987 auch im Westen. Seine Arbeit über die sonografische Diagnostik der CholedochusSteine in unserer „Ultraschall in der Medizin" ist heute noch bester sonografischer Standard. In dieser Zeit habilitierte er sich über Portale Hypertonie und Dopplerdiagnostik.

Wolfram wusste in der „Wendezeit“ nicht, wie es für ihn und an der Charité weitergehen würde. Obwohl unbelastet, fürchtete er seine Stelle nicht behalten zu dürfen. Die Bürokratie arbeitete langsam aber schließlich bekam er seine Professur. Die Klinik war unterbesetzt und er leistete viele zusätzliche Bereitschaftsdienste, und zwar unentgeltlich. Meinem Ratschlag, eine Stelle im Westen zu suchen, folgte er nicht.

Typisch Wolfram Wermke wollte er weder Klinik, noch Kollegen, noch Patienten im Stich lassen. Es war damals für ihn mit großer Familie nicht einfach bei den ständig steigenden Preisen und dem um $30 \%$ reduzierten Ost-Grundgehalt.

Er hielt die Abteilung für Gastroenterologie in der Charité zusammen und wartete hoffnungsvoll auf einen neuen Ordinarius. Wie in vielen anderen Bereichen auch lief es leider dann in der Folge doch nicht so glatt. Peu à peu wurde er aus der Klinik in die Sonografie abgedrängt. Keine einfache Situation für einen begeisterten Kliniker, gut je- doch für das, was er in der Sonografie in seinem weiteren Leben geleistet hat.

Wolfram ist ein Typ mit Ecken und Kanten, er ist brillant in seiner Arbeitsweise, hält exzellente bildreiche Vorträge - er und seine Vorträge sind mittlerweile Kult geworden. Wolfram hat immer viele Anekdoten parat, hier sei eine über seine Begegnung mit seinem großen Vorbild Frerichs, der als Begründer der Hepatologie gilt, kurz nacherzählt. In der Wendezeit fand er im Kohlenkeller der Charité Theodor Frerichs 1861 herausgegebene Originalpublikation „Klinik der Leberkrankheiten “ mit dem ex libris von Rudolf Virchow. Wolfram rettete das rare und bestens erhaltene Sammlerstück, kopierte es sorgfältig und gab das Buch zurück. Später wurde es in der Vitrine ausgestellt und - Ironie des Schicksals - irgendwann geklaut.

Frerichs exzellenter Darstellung der Morphologie der Lebertumoren und ihren sensationellen farbigen Abbildungen stellte Wolfram Ultraschallbilder als Pendant gegenüber und diese Vorträge machten Wolfram zu einem sehr begehrten Redner. Dies hätte alleine genügt um mit der DEGUMMedaille ausgezeichnet zu werden.

Sehr bald war Wolfram auch im Westen der Republik daher als Ultraschallexperte anerkannt und wurde von der Industrie immer mit den neuesten Geräten versorgt. Nicht wenige Kollegen waren neidisch, weil er bei Neueinführungen oft diese Geräte als erster bekam und diese mit vielen tollen Bildern vorstellen konnte. Er war ein Meister im Umgang mit Patienten und Geräten. Niemand verstand es wie er, so wunderschöne Ultraschallbilder aus Gerät und Patienten herauszukitzeln.

Wie er selbst sagte, habe er vieles seinem Freund und Physiker Bernhard Gaßmann zu verdanken. Sie hatten mehr als nur an den Reglern herumgeschraubt. So kam es, dass sich Wolfram Wermke extrem mit dem Farbdoppler und sehr früh mit Kontrastverstärkern beschäftigte. Bereits 1998 brachte er zusammen mit B. Gaßmann eine Monografie, die auch in Englisch erschien, wurde leider wenig nachgefragt, weil die Zielgruppe der Leser damals noch nicht reif für das Thema war. Noch heute stellt diese „Tumordiagnostik der Leber mit Echosignalverstärker" eine Fundgrube für Besonderheiten dar.

Auch wenn Wolfram Wermke von manchen in seinem Berliner Umfeld von manchen Kollegen gemieden oder gar "geschnitten“ wurde, ging er kontinuierlich und konsequent seinen Weg weiter. Er sah sich in der Tradition der alten Charité und ihrer großen Kliniker. Ihm ging fundierte Arbeit vor schnelles und unreflektiertes Publizieren.

Wolfram Wermke konnte sich gelegentlich drastisch ausdrücken und auch mal derb sein, jemandem nach dem Mund zu reden war seine Sache nie. Er zitierte lieber Max Liebermann: „Kunst kommt von können, käme es von wollen müsste es Wulst heißen“.

Vieles von dem, was er erarbeitete, publizierten seine Schüler, und so findet man in PubMed doch eine ganze Menge von ihm. Wolfram hatte Gott sei Dank ein paar „Eckermänner“.

Wolfram ist im besten Sinne ein sonografischer Dr. House, er ist ein Meister im Knacken harter Nüsse, nicht selten mithilfe einer vertieften Anamnese. Mit Hingabe verfolgt er lange Verläufe, um so Änderungen der Morphologie exakt zu erfassen. Er pflegt sein riesiges Ultraschallarchiv, keiner erreicht seine Fallzahlen und scheinbar nebenher recherchiert er ausgiebigst pathologische und anderweitige bildgebende Literatur. Daraus resultierte dann ein nach langjähriger Beschäftigung ein im Alleingang erstelltes fundiertes Werk, nämlich die „Sonografische Leberdiagnostik“.

Lieber Wolfram, Lehre und Wissensvermittlung waren dir stets wichtig. Wenn du auch das große Kongresstheater durchaus genieBen konntest, war dir ein Auftritt bei deinen Ultraschallfreunden im Kreis interessierter 
Kollegen viel lieber. Gerne führst du Diskussionen auf Augenhöhe, denn der wissenschaftliche Streit, die Keimzelle aller wissenschaftlichen Arbeit, benötigt fundierte Gesprächsrunden und vor allem auch Zeit. Mit deiner aktuellen „Interaktiven Veranstaltungsreihe" hast du ein neues Format erfunden. Wolfram und seine Sono-Schüler plaudern dort aus dem Nähkästchen, zeigen interessierten Kollegen, wie Sonografie geht, wie man sonografische Probleme löst, worauf man hereinfällt und wie man das vermeiden kann.

So pflanzt Wolfram unermüdlich sein fundiertes Wissen durch direkte und indirekte Weitergabe gleichsam metastatisch fort.

Wolframs Leben ist leider nicht ganz ohne Belastungen und Dellen geblieben, als Schwachstromelektriker hast du beruflich in der DDR begonnen, dagegen ist dein Leben immer hochvoltig verlaufen, Abhauen war deine Sache nicht, dafür hättest du - wie du sagtest - zu viele Geiseln hinterlassen.

Rettenmaier, der Wolfram ob seines Könnens und seiner fundierten wissenschaftlichen Statements sehr schätzte, stellte früh fest: Wolfram sei ein Typ, der seine Lebenskerze an zwei Enden gleichzeitig abbrenne. Immer wieder spielte ihm seine Gesundheit heftige Streiche, sodass er seine körperliche Belastbarkeit im Laufe der Zeit zurückfahren musste. Wenn man sieht, welches Quantum oder besser welches „Qualitativum" er dennoch bewältigte, so ist dies sicher auch seiner Ehefrau Martina zu verdanken, die ihn je nach Bedarf beschützt, ermuntert oder bestärkt.

Wolfram ist die sonografische Kapazität unserer Gesellschaft im Bereich der Inneren Medizin, der als fundierter Kritiker und
Querdenker von manchem Kollegen wahrgenommen oder sogar gefürchtet wird.

Wolfram Wermke hat sich immer als eigener Kopf erwiesen und sich deshalb mit der DEGUM als Institution immer etwas schwer getan, jedenfalls schwerer als die Gesellschaft mit ihm. Wenn auch nicht gänzlich uneitel fällt es ihm nicht leicht, öffentlich Ehrungen entgegenzunehmen. Wolfram ist Preuße durch und durch, seine Ideale findet man in der Historie der Charité, angefangen bei Schönlein, Virchow und Frerichs, und nicht zu vergessen medizinisch auch bei Professor Berndt, der ihm während der DDR-Zeit ein sehr guter klinischer Lehrer gewesen sei.

Die DEGUM ist stolz, dich heute im Kreise deiner Schüler zu ehren, dir danke ich für viele Fachgespräche und die langjährige Freundschaft!

Karlheinz Seitz 